\title{
Increased Respiratory Frequency and Variability in High Risk Babies Who Die of Sudden Infant Death Syndrome
}

\author{
DANIEL C. SHANNON, DOROTHY H. KELLY, SOLANGE AKSELROD, AND \\ KENNETH M. KILBORN \\ Pediatric Pulmonary Unit, Children's Service, Massachusetts General Hospital, Boston, Massachusetts 02114
}

\begin{abstract}
We have tested the hypothesis that autonomic instability, reflected in increased variability of heart rate and respiratory frequency, characterized high risk babies who died of sudden infant death syndrome. Using computer-based methods, we compared the power spectra of instantaneous heart rate and respiration on coded tape recordings from seven asymptomatic siblings and 10 babies with symptomatic apnea who died of sudden infant death syndrome to 34 age- and sex-matched controls. We confirmed our previous observation of increased respiratory bandwidth, an index of variability in respiratory frequency $(p=0.009)$ but failed to confirm our finding of increased low frequency fluctuations in heart rate $(p=0.18)$. In addition, we found an increase in mean respiratory frequency during quiet breathing $(p=0.001)$ and a significant relationship between respiratory bandwidth and mean respiratory frequency $(r=0.604, p=0.0002)$. These variables along with those from a previous analysis of the same data base yield a discriminant function with $82 \%$ sensitivity and $100 \%$ specificity. These results confirm previous suggestions that high risk babies who die of sudden infant death syndrome exhibit autonomic instability. (Pediatr Res 22: 158-162, 1987)
\end{abstract}

$\quad$ Abbreviations
RBW, respiratory band width
SIDS, sudden infant death syndrome
ROC, receiver operating curve
HR, heart rate
PB, periodic breathing
Hz, Hertz (cycles/s)

We have hypothesized that the at-risk state for sudden and unexplained death in infants (SIDS) is characterized by abnormal patterns of fluctuations in heart rate and respiratory activity (1, 2). In our first test of this hypothesis, we compared fluctuations in instantaneous heart rate and respirations using power spectrum analysis and identified two abnormalities that were statistically different in high risk babies who died of SIDS compared to controls (2). SIDS babies exhibited increased fluctuations in heart rate at frequencies of $0.02-0.10 \mathrm{~Hz}$ and increased variability of respiratory frequency. However, in a blinded trial of this hypothesis, using recordings obtained prospectively by Gordon et al. (3) on English babies, we were unable to identify SIDS

Received June 10, 1986; accepted February 26, 1987.

Address correspondence to Daniel G. Shannon, M.D., Pediatric Pulmonary Unit, Massachusetts General Hospital, Boston, MA 02114. babies among controls by either increased low frequency HR fluctuations or increased variability of respiratory frequency. We concluded that the differences between these two studies might be explained by differences in methods or most probably in the populations. In order to test the initial hypothesis, we have repeated the analysis of data from our high risk population using a larger, blinded and coded data set in order to avoid any possibility of bias. The coding was performed by a technician who was unaware of the past history of the subjects and of the results of a pneumogram analysis of recordings obtained with an independent software program (4). Using the latter program, we identified a combination of variables (mean heart and respiratory rates, episodes of bradycardia, chronologic age, and longest episode of periodic breathing) using discriminant analysis that identified $72 \%$ of SIDS with $2 \%$ false-positives.

\section{METHODS}

Subjects and data collection. From our clinical population, we identified 20 full-term babies who were unmedicated at the time of their pneumograms and who subsequently died of SIDS. Recordings on three infants were technically inadequate for analysis by the software program already described (4) because of excessive high frequency noise that affected the entire recording. The remaining 17 infants included seven asymptomatic siblings and 10 infants who had apnea (4). Only two of these 17 served as the basis for our preliminary report of power spectrum abnormalities (2); the other six from that report were excluded because of the coexistence of seizures or gastroesophageal reflux (three babies) or noisy recordings (three babies). We have previously excluded from the symptomatic group those identified to have causes of pathologic apnea treatable by standard clinical methods. Recordings on controls were obtained as part of a prospective data collection from infants born in a local obstetric unit. They were chosen at random from our files; if a recording was technically inadequate for computer analysis, another ageand sex-matched recording was chosen at random. All recordings were coded so that technicians and investigators were blinded to the origin of each tape cassette. Biographic data on controls and SIDS are in Table 1. Their weights were comparable; sex ratios were equal. As part of the initial evaluation we obtained pneumogram recordings (5) on all infants. These recordings and those obtained at home on 34 healthy unmedicated infants (two controls for each SIDS baby) were matched for sex and chronologic age.

Data sampling. We used our method of power spectrum analysis originally described in animals (6) and subsequently applied to humans $(2,3,7)$. Recorded data were selected for power spectrum analysis as described (2). Briefly, all segments of at least $5 \mathrm{~min}$ of quiet breathing were identified visually from the 12-h analog recordings. We excluded segments with sighs, 
Table 1. Characteristics of study infants

\begin{tabular}{lccc}
\hline & $\begin{array}{c}\text { SIDS } \\
(n=17)\end{array}$ & $\begin{array}{c}\text { Controls } \\
(n=34)\end{array}$ & $p$ value* \\
\hline Birth wt (kg) & $\begin{array}{c}3.28 \pm 0.49 \dagger \\
(2.7-4.1)\end{array}$ & $\begin{array}{c}3.45 \pm 0.49 \\
(2.6-4.6)\end{array}$ & 0.21 \\
& $\begin{array}{c}39.76 \pm 1.48 \\
(37.5-43)\end{array}$ & $\begin{array}{c}40.09 \pm 0.79 \\
(38-42)\end{array}$ & 0.35 \\
Gestational age & $(w k)$ & & \\
$\quad$ Chronologic age & & & \\
$\quad$ At recording (wk) & $5.73 \pm 6.01$ & $5.89 \pm 5.59$ & 0.53 \\
& $(0.3-21.1)$ & $(1.3-21.1)$ & \\
At death (wk) & $15.80 \pm 14.27$ & & \\
& $(3.4-45.4)$ & & \\
Sex & & 18 & \\
$\quad$ Male & 9 & 16 & \\
Female & 8 & & \\
\hline
\end{tabular}

* Student's $t$ test, 2-sided.

$\dagger$ Mean \pm SD (range).

apnea, bradycardia, or tachycardia, and artefacts. The corresponding segments on the magnetic tape cassettes were then transferred to FM tape for slower replay from which they were sampled through a phase-locked loop frequency multiplier that locked onto the respiratory carrier signal $(22 \mathrm{~Hz})$ used to record the respiratory waveform (8). This was done in order to eliminate fluctuations introduced by either the recording unit (Oxford Medilog Recorder) or the replay module (Oxford PMD $12 \mathrm{Re}$ play Unit). Respiratory signals and ECG were sampled at a frequency of $360 \mathrm{~Hz}$ from the FM tape played at real time. A peak detection algorithm was used to identify each $R$ wave; the time series of RR intervals and the respiratory wave forms were then sampled at equal time intervals $(4 \mathrm{~Hz})$ having been passed through a $2 \mathrm{~Hz}$ antialiasing filter. The latter avoids inaccurate frequency determination that can be introduced by spectral analysis if the sampling frequency does not exceed twice the highest frequency component of the signal (Nyquist frequency).

Spectral analysis. As described in greater detail in previous publications $(2,3,7)$, power spectrum analysis was chosen because it characterizes the distribution of variations about the mean as a function of frequency. The frequencies at which variations occur result from the differing response times of various autonomic controllers and the power at these frequencies is an estimate of the original noise and the loop gain of the controlled systems where loop gain reflects the ratio of output to input. Since we lack quantitative information about the input, differences in power must be interpreted with caution because they can reflect differences in either input (magnitude of noise or perturbation) or output (gain in the autonomic controlled system).

Power spectra were calculated from the time series of instantaneous HR and respiration using a discrete Fourier Transform. From each spectrum of instantaneous HR we estimated the magnitude of low and high frequency power (power = amplitude $^{2}$ ). Low frequency power was defined as the integral of power from $0.02-0.10 \mathrm{~Hz}$, high frequency power was defined as the integral of power in the band width that corresponded to the band width of the respiratory power spectrum (2). From prevoius studies on healthy adults, we have found that both of these measures mainly reflect parasympathetically mediated modulation of HR when measured in supine position, the high frequency peak being purely parasympathetic and the low frequency being partly sympathetic (7). From the power spectrum of the respiratory signal, we measured the RBW as described previously (2). Although this method results in a subjective estimate of the frequencies above and below which the signal is only noise, it should not systematically bias a blinded study. In addition to measuring the spectral content of the RR interval time series and respiratory waveform, we measured the mean values of $H R$ and respiration.
Normalization. As in previous investigations, we normalized the HR spectrum by the square of the mean HR in order to yield a dimensionless variable (amplitude ${ }^{2} /$ mean $\mathrm{HR}^{2}$ ). From a physiologic as well as mathematic perspective this appears attractive because increased $\beta$ adrenergic activity increases both mean HR as well as the variability at low frequency (6); on the other hand increased parasympathetic activity lowers mean rate while increasing variability. In the present investigation, we examined the normalized spectra so that they could be compared to those in our previous work. We also examined the results without normalization and found (vide infra) that we could not reject the null hypothesis in either case. The respiration waveform was analyzed as a continuous variable and therefore was not normalized. The spectral content of the fundamental peak (RBW) reflects mainly the variability of breath to breath intervals, but also may be affected by periodic fluctuations in either breath amplitude or frequency. In general, a broader fundamental peak in the respiratory band can be interpreted as greater breath-tobreath variability or as periodic amplitude or frequency modulation. In the latter case, the peak will be widened by twice the frequency of the periodic fluctuation and there will be an additional peak at the frequency of the periodic fluctation.

Stationarity criteria. In order to maximize reliability of power spectrum analysis, we developed criteria of stationarity for both the instantaneous HR and respiratory waveforms. Software was developed to calculate the mean and variance of each of six segments of equal duration for each 256-s time series. The technician provided us with a subset of 55 traces from control subjects, blinded with respect to the total data set. From 330 segments of these traces, we generated values for the relative deviation of each of four parameters: mean HR and variance, mean respiratory frequency and variance where relative deviation was defined as $[\bar{x}($ segment $)-\bar{x}($ trace $)] / \bar{x}($ trace $)$. The upper 2.5 percentile limit for each of these four distributions was subsequently used as our limit of stationarity. We then analyzed all data using these new quantitative criteria. If, in any subtrace of a subsequently studied tracing, the computed relative deviation of any of the four parameters exceeded this definition of stationarity, the entire tracing was excluded from further analysis. Note that although obvious examples of periodic breathing had been excluded by visual inspection, subtle examples may remain and, if continuous for the 256-s time series, would remain after the stationarity criteria were applied.

From the 51 recordings, only 36 had at least one quiet breathing segment suitable for analysis. While the elimination of these 15 recordings might theoretically bias the results, 10 were controls and five were SIDS cases and the 2:1 ratio of controls to cases was preserved in the final analysis. In the remaining 36 recordings, we identified between one and 13 segments for sampling. In order to avoid bias introduced by including unequal numbers of segments from each baby, we selected for comparison the one segment for each baby that contained the least low frequency power in the HR spectrum as in previous studies (2, 3 ). We chose this method because undetected unstationarity in the time series would most likely artifact the low frequency power; thus, this approach improved upon the visual estimate of stationarity.

Rank ordering and identification procedure. All measured variables extracted from power spectrum analysis were then rank ordered for those segments defined as stationary by eye ( 36 babies) and by quantitative criteria ( 34 of these 36 babies). We were advised by the technician who held the code that these study populations included 12 and 11 SIDS babies, respectively. Thus, the quantitative criteria for stationarity eliminated two time series as unstationary, one from a control and one from a SIDS case. Based on the results of our previous study (2) we expected to find in the data set selected by visual inspection that after rank ordering a significant fraction of SIDS cases would be included in the 12 babies with the most low frequency power in the HR spectrum and in the 12 with the broadest RBW. Similarly 
for the data set selected by quantitative stationarity criteria, we expected that most SIDS cases would be found in the top 11 babies. (Of the 11, six came from the apnea of infancy group and five from the asymptomatic siblings). Having thus rank ordered the entire population according to low frequency power and RBW with equal weight given for each of these two variables, we then broke the code. We used the Mann-Whitney $U$ test to test the null hypothesis that the distribution of SIDS cases in the rank order of low frequency HR power and RBW did not differ from controls.

With the code broken, we used Fischer's exact test to calculate the significance of the differences in proportions of SIDS compared to controls in the top 12 (stationary by eye) and top 11 (stationary by computer analysis), respectively. Since the variables measured were not normally distributed we compared the medians of all variables using the two-tailed Wilcoxon-MannWhitney rank sum test. We accepted a result as significantly different if the null hypothesis was excluded at a $p$ value $=1-$ $\sqrt[n]{0.95}$ in order to account for the effect of multiple comparisons. Since we compare low frequency power, mean HR, mean RR and RBW, $n=4$, and thus $p=0.013$.

Finally, in order to identify the discriminant power of these observations plus those from a parallel and independent investigation of the same population of babies (4), we entered into a single computer data base the values of each measured variable in the present study along with the variables from the previous study. This previous investigation extracted from each recording the mean $\mathrm{HR}$, mean respiratory rate, $\%$ periodic breathing, apnea density, bradycardia, tachycardia, and quiet and active time for the entire $12-\mathrm{h}$ recording. We next identified the variables that were significantly different (SIDS versus controls) at $p<0.15$ using these variables and those from the present study using canonical discriminant analysis (SAS) which generated the weighted sum of these variables; the computer identified the combination of five variables which maximized the separation (Mahalanobis distance) of SIDS from controls, the discriminant function. We then generated a ROC for the discriminant function which plots true versus false positive identification for chosen threshold levels.

\section{RESULTS}

The rank order of controls and SIDS, equally weighted for the values of RBW and the power of HR oscillations at low frequency, failed to identify SIDS cases at a rate greater than chance. This failure occurred because high values for low frequency power were proportionately distributed between SIDS and controls. However, a rank order of RBW alone identified the SIDS cases at a rate significantly greater than chance $(p=0.013)$. These observations refute our previous suggestion that low frequency HR power is associated with SIDS but support the relationship between SIDS and RBW. Having broken the code, we examined the absolute values for the measured variables and the relationship between RBW and respiratory frequency.

The respiratory bandwidth was $0.165 \mathrm{~Hz}(\mathrm{SD}=0.046)$ in SIDS and $0.117 \mathrm{~Hz}(\mathrm{SD}=0.048)$ in controls, a difference that is significant at $p=0.009$. The power of HR oscillations at low frequency was $7.65 \times 10^{-5}\left(\operatorname{SD} 6.7 \times 7 \times 10^{-5}\right)$ in SIDS and 5.94 $\times 10^{-5}\left(4.8 \times 10^{-5}\right)$ in controls $(p=0.18)$ (Table 2). These observations confirm those from the blinded study of rank order.
Mean respiratory frequency was higher in SIDS, 43.8 breaths per min $(0.73 \pm 0.14 \mathrm{~Hz})$ than controls 34.2 breaths per min $(0.57 \pm 0.11 \mathrm{~Hz}), p=0.001$. Furthermore, mean respiratory frequency varied directly with respiratory bandwidth $(r=0.604$, $p=0.0002$ ) (Fig. 1). The SIDS babies clustered at the upper end of the regression and the slope of the regression was significantly different from zero for the controls alone $(p=0.009)$ but not for the SIDS babies alone $(p=0.22)$. Significant differences were similarly obtained for the data selected for stationarity by eye (36 babies) and by computer method ( 34 babies). Thus, the software for stationarity criteria improved the quantitative rigor of the analysis but did not improve the ability to separate SIDS from controls. We then segregated the six babies with apnea of infancy and the five who were asymptomatic siblings. Although the differences in RBW and mean respiratory frequency were similar for each subgroup compared to controls only the difference in RBW in asymptomatic siblings reached statistical significance using a threshold $p$ value of 0.013 that accounts for multiple comparisons (Table 3).

Discriminant analysis, incuding the variables from our previ-

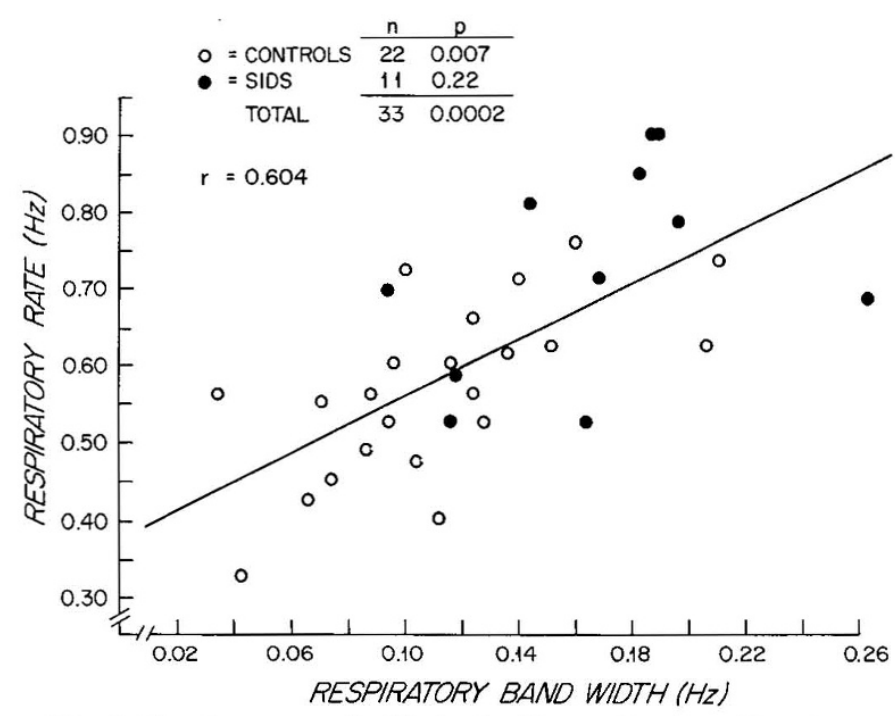

Fig. 1. Respiratory rate in $\mathrm{Hz}$ (cycles/s) as a function of respiratory band width in $\mathrm{Hz}$ for a 256-s data segment on each of 22 controls $(\mathrm{O})$ and 11 SIDS (๑). These data include only those who passed the quantitative criteria for stationarity. The regression line with a correlation coefficient of $r=0.604$ was significantly different from zero at $p=0.002$. A regrussion for the controls alone $(p=0.007)$ was also significant while that for SIDS alone was not.

Table 3. Respiratory parameters in apnea of infancy cases and asymptomatic siblings of SIDS compared to controls

\begin{tabular}{lcccc}
\hline & $\begin{array}{c}\text { Apnea of } \\
\text { infancy } \\
(n=6)\end{array}$ & $p^{*}$ & $\begin{array}{c}\text { Asymptomatic } \\
\text { siblings } \\
(n=5)\end{array}$ & $p^{*}$ \\
\hline RR & $0.74 \pm 0.16 \mathrm{~Hz}$ & 0.038 & $0.71 \pm 0.13 \mathrm{~Hz}$ & 0.048 \\
RBW & $0.14 \pm 0.04 \mathrm{~Hz}$ & 0.236 & $0.19 \pm 0.04 \mathrm{~Hz}$ & 0.006 \\
\hline
\end{tabular}

${ }^{*} p$ values (Wilcoxon) compared to controls (see Table 2 ).

Table 2. HR and respiratory parameters in SIDS babies compared to controls

\begin{tabular}{lccc}
\hline & $\begin{array}{c}\text { Controls } \\
(n=23)\end{array}$ & $\begin{array}{c}\text { SIDS } \\
(n=11)\end{array}$ & $\begin{array}{c}p \text { value } \\
\text { Wilcoxon }\end{array}$ \\
\hline H/min & $130.7 \pm 15.5$ & $139.3 \pm 12.3$ & 0.14 \\
HR power & $5.94 \times 10^{-5} \pm 4.8 \times 10^{-5}$ & $7.65 \times 10^{-5} \pm 6.7 \times 10^{-5}$ & 0.18 \\
RR (Hz) & $0.57 \pm 0.11$ & $0.73 \pm 0.14$ & 0.001 \\
RBW $(\mathrm{Hz})$ & $0.117 \pm 0.05$ & $0.166 \pm 0.05$ & 0.009 \\
\hline
\end{tabular}




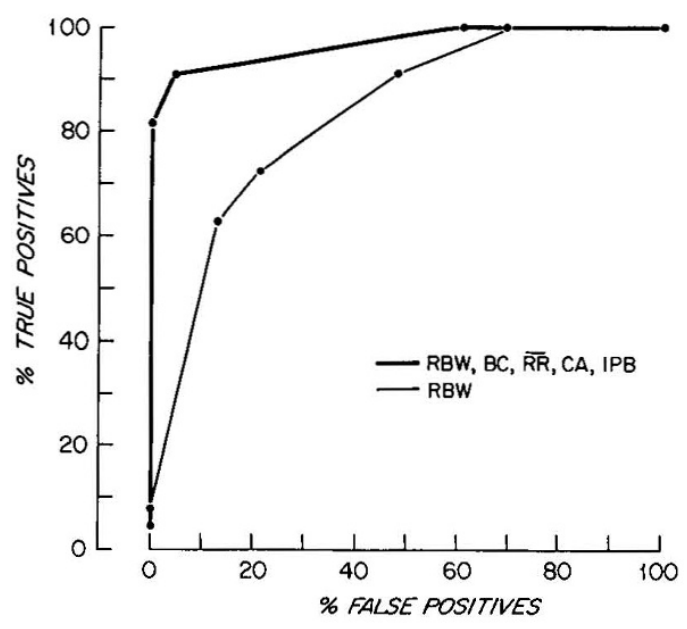

Fig. 2. The ROC showing the true-positive identification of SIDS cases at arbitrarily chosen threshold values of false-positive rates. The heavy solid line shows the best identification of SIDS cases with $82 \%$ true-positive and no false-positives. This curve was generated by computer using discriminant analysis (SAS). The optimal five variables were the RBW and mean RR from the present investigation as well as the incidence of bradycardia $(B C)$ and longest episode of periodic breathing $(L P B)$ from a previous investigation using all $12 \mathrm{~h}$ of recorded data from each baby as well as chronologic age $(C A)$ common to both studies. An ROC curve for RBW alone (thin solid line) is shown for comparison.

ously published pneumogram study on the identical population of SIDS cases and controls (4) yielded a ROC curve that identified $82 \%$ of SIDS (true-positive) with no false-positives (Fig. 2). This curve included the respiratory bandwidth which accounted for the most discrimination of a single variable along with respiratory frequency from the current investigation and three variables derived from analysis of the entire 10-h long pneumogram recording from the previous investigation: episodes of bradycardia, chronologic age, and the longest episode of $\mathrm{PB}$. This result improved substantially the previous discriminant function obtained solely from the pneumogram parameters (4) that yielded a $6.5 \%$ false-positive identification at the same true-positive rate (4).

\section{DISCUSSION}

The results of this investigation confirm those of our preliminary spectral analysis study (2) in identifying increased respiratory bandwidth during quiet breathing as a parameter that separates SIDS babies from controls; however, they failed to confirm the previous observation of increased low frequency HR variability as a second parameter. We have extended our previous observations by identifying significantly increased mean respiratory rate in SIDS babies compared to controls. We also found a strong positive correlation between RBW and mean respiratory frequency. Finally, when the present results are added to those of a previous investigation of the same population of both SIDS and controls (4), a discriminant function is derived that yields $82 \%$ sensitivity and $100 \%$ specificity in separating high risk SIDS from controls. This discriminant function is based on RBW and mean respiratory frequency from the present investigation and on the frequency of episodes of bradycardia, the longest episode of $\mathrm{PB}$ during the entire 12-h recording from the parallel investigation (4), as well as chronologic age. The most discriminating parameter was RBW (Fig. 2).

Increased respiratory bandwidth probably arose from more variability in breath to breath intervals in SIDS babies. Periodic fluctuations in frequency can add side lobes to the fundamental peak separated by the frequency of the period and, thus, broaden the RBW; however, we found no increased power at the fre- quency of PB $(0.02-0.07 \mathrm{~Hz})$ in the respiratory spectrum that would support this explanation.

We observed among the SIDS infants higher respiratory frequencies in the 5-min segment of quiet breathing. From clinical data collected when the pneumograms were performed, acidemia, hypoxemia, anemia, and high surface to mass ratios (increased metabolic rate) can be excluded. Among other possible causes would be a systematically low ambient temperature, increased dead space ventilation, or increase $\beta$ adrenergic activity. None of these possibilities can be excluded.

The exclusion criteria used in the present investigation eliminated six of eight SIDS cases that were included in our preliminary report of power spectrum abnormalities (2). These cases contributed the high levels of low frequency HR power found in that study. Thus, their exclusion accounts for our failure to confirm that observation in the present study. The increased power may have resulted from the clinical conditions, (seizures, gastroesophageal reflux), from medications (anticonvulsants, metaclopramide), or most probably from unstationary time series all of which were excluded in the present investigation.

The present results are different from those that we obtained from British babies recorded by Southall et al. (10). The explanation for these differences probably lies in the study populations rather than in the methods. Our current population included 10 infants with symptomatic apnea and seven asymptomatic siblings while the British population was selected from full-term births in three separate obstetric departments. Differences in the methods of obtaining respiratory signals included different transducers and different filtering by the preamplifiers. Neither of these differences should systematically affect respiratory frequency. Thus, we believe that the difference in physiology most likely arose because of differences in subject selection.

Finally, we have found that a discriminant function that includes RBW, mean respiratory frequency, the longest episode of periodic breathing, bradycardia and chronologic age identifies $82 \%$ of SIDS with no false-positives. This analysis excluded several variables that, individually, were significantly increased in SIDS compared to control infants; these were the \% time in $\mathrm{PB}$ and the mean HR over the entire 12-h recording. This exclusion occurs when the computer finds an optimal combination and probably reflects similar discriminating abilities between an included (longest episode of PB) versus excluded (\% PB) variable. We emphasize that we were not prospectively testing this population using this discriminant function and, therefore, the results must be viewed with caution appropriate to a retrospective analysis.

Our observations support the hypothesis that SIDS in high risk babies is associated with defective regulation of vital functions. However, further studies involving high risk survivors are necessary in order to understand the biologic significance of our observations. Increased variability of respirations might reflect an inherent instability in feedback and control mechanisms which at times results in periodic apnea; such instability, however, may not be a sufficient condition to explain death.

\section{REFERENCES}

1. Kelly D. Shannon DC 1981 Treatment of apnea and excessive periodic breathing in the full term infant. Pediatrics 68:183-186

2. Gordon D, Cohen R, Kelly DH, Akseirod S, Shannon DC. 1984 Sudden infant death syndrome: abnormalities in short term fluctations in heart rate and respiratory activity. Pediatr Res 18:921-926

3. Gordon D. Southall DP. Kelly DH, Wilson A, Akselrod S, Richards J, Kenet B, Kenet R, Cohen RJ, Shannon DC 1986 Analysis of heart rate and respiration of sudden infant death syndrome (SIDS) victims and control infants. Pediatr Res 20:680-684

4. Kelly DH, Golub H, Carley D. Shannon DC 1986 Pneumograms in infants who subsequently died of SIDS. J Pediatr 109:249-254

5. Stein IM. Shannon DC 1975 The pediatric pneumogram-a new method for detecting and quantitating apnea in infants. Pediatrics 5:589-594

6. Akselrod S, Gordon S. Madwed JB, Snidman NC, Shannon DC. Cohen RJ 1985 Hemodynamic regulation: investigation by spectral analysis. Am J Physiol 249(Heart Circ Physiol (18):H867-H875 
7. Pomeranz B, Macaulay RJB, Caudill MA, Kutz I, Adam D, Gordon D, Kilborn KM, Barger AC, Shannon DC, Cohen RJ, Benson H 1985 Assessment of autonomic function in man by heart rate spectral analysis. Am J Physiol 248(Heart Circ Physiol 17):H151-H153

8. Wodicka GR, Shannon DC, Burns SK 1986 A flutter compensation scheme for tape recorded physiologic signals. Proceedings AAMI 21st Annual Meeting
9. Cooley WW, Lohnes PR 1971 Multivariate Data Analysis. New York, Wiley 10. Southall DP, Richards JM, de Swiet M, Arrowsmith WA, Cree JE, Fleming PJ, Franklin AJ, Orme RL'E, Radford MJ, wilson AJ, Shannon DC, Alexander DR, Brown NJ, Shinebourne EA 1983 Identification of infants destined to die unexpectedly during infancy: evaluation of predictive importance of prolonged apnea and disorders of cardiac rhythm on conduction. Br Med J 286:1092-1096

\section{Announcements}

\section{European Congress of Perinatal Medicine}

The Congress will take place on April 10-13, 1988 at the Hilton International Hotel, Rome, Italy.

The Congress will include plenary sessions devoted to congenital abnormalities, perinatal nutrition and its derangements, and perinatal infections. Seminar topics will include: fetal and neonatal endocrinology, fetal and neonatal neurosensorial development, perinatal respiration, embryo and fetal manipulation, perinatal pharmacology and pharmacodynamics, perinatal cardiovascular pathophysiology, management of the small premature infant, management of hypertension in pregnancy, and new technologies. Workshops will include sessions devoted to work and pollution, perinatal pathology, perinatal bioengineering, and emerging areas. Official language for the Congress will be English. Simultaneous translation will be provided according to the number of participants for each additional language.

Deadline for abstracts and early registration: November 1, 1987.

President: Professor Ermelando V. Cosmi. Scientific Secretariat: Dr. G. C. Di Renzo, Via degli Olivi 72, 06100 Perugia, (75) 62552/29271. Organizing Secretariat: Wagons-lits Turismo, Congress Department, Via Abruzzi 3, 00187 Rome, (6) 4757651/4, telex: 611504 Walbo I. 